\title{
RESPUESTA DE LA CANOLA AL DÉFICIT HÍDRICO DEL SUELO
}

\author{
CANOLA RESPONSE TO SOIL WATER DEFICIT
}

\author{
Marco A. Inzunza Ibarra*, Ernesto A. Catalán Valencia, Magdalena Villa Castorena, \\ Ignacio Sánchez Cohen y Abel Román López
}

\begin{abstract}
Centro Nacional de Investigación Disciplinaria en Relación Agua Suelo Planta Atmósfera (CENID RASPA), Instituto Nacional de Investigaciones Forestales, Agrícolas y Pecuarias (INIFAP). Canal Sacramento km 6.5, margen derecha. 35140, Gómez Palacio, Dgo., México. Tel. y Fax 01(871)1590104, 05 y 07. Fax Ext. 106.
\end{abstract}

* Autor para correspondencia (inzunza.marco@inifap.gob.mx)

\section{RESUMEN}

El trabajo se llevó a cabo en Gómez Palacio, Durango, México, con el objetivo de determinar la función de producción que relacione el rendimiento de canola (Brassica napus L.) con el nivel de humedad del suelo. Se aplicaron siete tratamientos de riego, consistentes de seis diferentes niveles de abatimiento de la humedad aprovechable residual del suelo al momento de regar $(0,12,24,36,48$ y $60 \%)$, y un tratamiento adicional con sólo el riego de presiembra. Se utilizó un diseño experimental de bloques completos al azar con cuatro repeticiones. Las variables evaluadas fueron rendimiento de grano, eficiencia del uso de agua y lámina de agua consumida. Según la función de producción obtenida, es posible lograr un rendimiento de grano de $3.1 \mathrm{t} \mathrm{ha}^{-1}$, con una lámina de agua de $48 \mathrm{~cm}$ distribuida con riegos aplicados cuando la humedad aprovechable residual en el suelo baja a $35 \%$, equivalente a una tensión de humedad de $\mathbf{- 0 . 7 4}$ MPa.

Palabras clave: Brassica napus, función hídrica de producción, régimen de humedad del suelo, lámina de agua consumida.

\section{SUMMARY}

This work was carried out at Gómez Palacio, Durango, México. The goal of the study was to find a production function for relating canola (Brassica napus L.) yield with soil water content. Seven irrigation treatments were applied, of which six were levels of residual available soil moisture depletion $(0,12,24,36,48$ and 60 $\%$ ), and a treatment of one irrigation applied before sowing. The experiment was a complete randomized block design with four replications. The measured variables were grain yield, water use efficiency and water crop consumption. According to the production function obtained, it is possible to reach a grain yield of $3.1 \mathrm{t} \mathrm{ha}^{-1}$, with a water depth of $48 \mathrm{~cm}$ by irrigating at $35 \%$ of residual soil available water, equivalent to a soil water tension of $-\mathbf{0 . 7 4} \mathrm{MPa}$.

Index words: Brassica napus, water production function, soil water regime, water crop consumption.

\section{INTRODUCCIÓN}

El cultivo de canola (Brassica napus L.) ha tenido gran aceptación mundial debido a su aceite de alta calidad para consumo humano y por adaptarse a temperaturas frías en su etapa de emergencia a roseta, aunque sus temperaturas óptimas son de 20 a $25{ }^{\circ} \mathrm{C}$ durante el ciclo (Gan et al., 2004; Villa et al., 2007). Por esta razón, Canadá es el principal productor de esta planta en el mundo, seguido por Australia y países de la Comunidad Europea (Rife y Zeinali, 2003). Actualmente la canola se produce en 40 países y ocupa un lugar importante por su contribución a la producción mundial de aceites vegetales. A nivel global se cosecharon 37060 millones de toneladas de grano por año durante el periodo 1997-2002, con un rendimiento promedio de $1.5 \mathrm{t} \mathrm{ha}^{-1}$ (Willenborg et al., 2004). En México, la producción de especies oleaginosas es insuficiente, ya que la industria aceitera importa cada año cerca de un millón de toneladas.

La canola es un cultivo de doble propósito porque además de producir aceite de alta calidad para consumo humano, produce forraje con mayor contenido nutricional que el de otros cultivos como trigo (Triticum aestivum L.) y algodón (Gossypium hirsutum L.). Asimismo, presenta ventajas adicionales como bajo consumo de agua, ciclo vegetativo más corto que otros cultivos oleaginosos como el cártamo (Carthamus tinctorius L.), y que al igual que éste tiene raíz de tipo pivotante que le permite aprovechar el agua del suelo de capas más profundas y ser más eficiente en el uso del agua (Johnston et al., 2003). La canola presenta bajas tasas de evapotranspiración como consecuencia a su alta capacidad de autorregulación (AlGhobari, 2000), por lo que es un cultivo deseable en regiones con baja disponibilidad de agua (Gan et al., 
2004), las cuales en México abarcan $97 \%$ de la superficie total (Sánchez, 2005).

Una estrategia para enfrentar el problema de la escasez de agua en las regiones áridas del país, es la diversificación del patrón de cultivos. Con ésta se pretende incluir cultivos de bajo consumo de agua y alta eficiencia de transformación (producción de materia seca por unidad de agua consumida). En este sentido, un cultivo alternativo con potencial es la canola, que en los últimos años ha interesado a agricultores, técnicos e investigadores por sus cualidades y porque su demanda se ha incrementado a nivel nacional y mundial, tanto en el ramo alimenticio (humano y animal) como en la industria de lubricantes y artículos de limpieza.

Para incluir una nueva especie en el patrón de cultivos es fundamental conocer sus necesidades hídricas y la eficiencia con que dicha especie utiliza el agua, es decir, la relación entre su rendimiento y la cantidad de agua consumida (Kassam et al., 2007). Esta información es necesaria para la planeación y toma de decisiones sobre el uso de los recursos hídricos a nivel regional. Por ejemplo, para optimizar el patrón de cultivos y definir las superficies por establecer de cada uno con la mayor rentabilidad posible, con base en el volumen de agua disponible y otras restricciones económicas, ecológicas y sociales (Sánchez et al., 2006).

El presente estudio se planteó con el objetivo de determinar una función de producción que relacione el rendimiento de canola con el nivel de humedad del suelo y la cantidad de agua consumida por el cultivo, mediante modelos que por su grado de generalidad podrían extrapolarse a otras regiones con condiciones climáticas similares.

\section{MATERIALES Y MÉTODOS}

El presente experimento se llevó a cabo en el ciclo agrícola otoño-invierno 2004-2005 en la Comarca Lagunera en el municipio de Gómez Palacio, Durango. El sitio experimental se localiza entre las coordenadas geográficas $25^{\circ} 31^{\prime} 57^{\prime}$ ' LN y $103^{\circ} 25^{\prime} 57^{\prime \prime}$ LO, a una altitud de $1139 \mathrm{~m}$, en la parte suroeste de Coahuila y noreste de Durango (Mendoza et al., 2004).

El clima de la Comarca Lagunera es de tipo desértico, muy árido y cálido, con escasa humedad atmosférica, precipitación pluvial anual promedio de $250 \mathrm{~mm}$ en la mayor parte de la región y de $450 \mathrm{~mm}$ en la zona montañosa oeste, con evaporación media anual de 2600 $\mathrm{mm}$ y temperatura media anual de $22{ }^{\circ} \mathrm{C}$. En este último aspecto, el área de la llanura y gran parte de la zona montañosa presenta dos periodos bien definidos: el primero comprende de abril a octubre con temperaturas medias mensuales mayores que $20{ }^{\circ} \mathrm{C}$, y el segundo de noviembre a marzo con temperaturas de 13.6 a $19.5{ }^{\circ} \mathrm{C}$. Los meses más fríos son diciembre y enero, y en este último mes se registra la temperatura media mensual más baja de $18{ }^{\circ} \mathrm{C}$. El periodo libre de heladas se ubica de abril a octubre, y pueden presentarse granizadas durante mayo. Las lluvias son en verano, con lluvias invernales que representan de 5 a $10.2 \%$ del total anual (García, 1970).

Las características físicas y químicas del suelo del sitio experimental (Cuadros 1 y 2) se determinaron en seis sitios de muestreo o repeticiones y tres profundidades del suelo por sitio $(0-30,30-60$ y $60-90 \mathrm{~cm})$, de las cuales se extrajeron muestras de $1 \mathrm{~kg}$ de suelo. Las características químicas medidas fueron los contenidos de: nitratos (método de Kjeldahl), fósforo (método de Duval), potasio (espectrofotometría), y carbonato total (volumetría), así como la conductividad eléctrica (conductimetría), porcentaje de sodio intercambiable y relación de adsorción de sodio (RAS). Las características físicas medidas fueron los contenidos de: materia orgánica (método de Walkley y Black), arena, limo y arcilla (método de la pipeta de Robinson), así como textura (triángulo de texturas), capacidad de campo (CC), punto de marchitez permanente (PMP) (método de la olla y membrana de presión) y densidad aparente (método del cilindro) (Castellanos et al., 2000; Richards, 1977).

Según estos análisis, el suelo contiene bajos niveles de nitrógeno total, es moderadamente bajo en fósforo y con altos contenidos de potasio, bajos contenidos de sales solubles, de sodio y de materia orgánica, según los rangos reportados por Castellanos et al. (2000). Con base en estos datos y la dosis de fertilización recomendada, se calculó y programó la fertilización del cultivo; con los valores de CC y PMP, que determinan la humedad aprovechable, se calculó y programó el riego de la canola.

Los tratamientos de riego consistieron en la aplicación de siete diferentes niveles de abatimiento de la humedad aprovechable residual en los primeros $90 \mathrm{~cm}$ de profundidad del suelo: $0,12,24,36,48$ y $60 \%$; se agregó un tratamiento adicional de sequía extrema, que consistió en aplicar únicamente el riego de presiembra (Cuadro 3). Los tratamientos se distribuyeron de manera aleatoria en el campo, con base en un diseño experimental de bloques al azar con cuatro repeticiones. Los bloques fueron colocados en forma perpendicular a la pendiente dominante del terreno, con el fin de disminuir esta fuente de error. A cada parcela o unidad experimental se le asignó una superficie de $20 \mathrm{~m}^{2}(4 \mathrm{~m}$ de ancho por $5 \mathrm{~m}$ de largo). Para determinar el contenido de agua del suelo y 
su variación a través el ciclo del cultivo, se aplicó el método gravimétrico en muestras de suelo tomadas con barrenas tipo Vehimeyer. Mediante la olla y la membrana de presión se obtuvieron las curvas características de humedad del suelo a tres profundidades, y se calcularon los valores de tensión de humedad de los tratamientos estudiados (Cuadro 4).

La siembra de canola se hizo el 16 de diciembre con el híbrido 'Hyola-401', a una densidad de siembra de $2 \mathrm{~kg}$ $\mathrm{ha}^{-1}$ en surcos de $80 \mathrm{~cm}$ de ancho; la separación entre plantas fue de aproximadamente $5 \mathrm{~cm}$ para tener una población de 250 mil plantas por hectárea. Con base en el muestreo inicial de suelos, se fertilizó con la fórmula $120 \mathrm{~N}-60 \mathrm{P}-00 \mathrm{~K}$, y la mitad del fertilizante se aplicó al momento de la siembra y el resto en el primer riego de auxilio.

Para regar el área experimental se instaló tubería presurizada de $15.2 \mathrm{~cm}$ de diámetro para conducir el agua desde la fuente de abastecimiento. También se instalaron hidrantes cada $36 \mathrm{~m}$ a lo largo de la tubería de conducción para conectar la tubería de compuertas con que se regó el área adyacente al experimento. Para regar las parcelas experimentales se utilizó tubería de $38.1 \mathrm{~mm}$ de diámetro, con conexiones para la unión de una manguera y un medidor volumétrico calibrado con el cual se aplicó de manera precisa el volumen de agua requerido.

Se hicieron muestreos de humedad del suelo al menos cada tercer día durante todo el ciclo del cultivo. El primer muestreo sirvió para conocer el contenido de agua del suelo antes del riego de presiembra, y el resto para monitorear el abatimiento de la humedad del suelo de cada tratamiento a través del ciclo del cultivo. Cada tratamiento se regó cuando el abatimiento de la humedad del suelo alcanzaba el nivel de abatimiento máximo predefinido para cada uno de ellos. En cada riego se aplicó la cantidad de agua necesaria para reponer la humedad del suelo hasta capacidad de campo en los primeros $90 \mathrm{~cm}$ de su profundidad.

Cuadro 1. Características químicas del suelo del sitio experimental.

\begin{tabular}{cccccccc}
\hline $\begin{array}{l}\text { Prof. } \\
(\mathrm{cm})\end{array}$ & $\begin{array}{c}\mathrm{NO}_{3} \\
\left(\mathrm{mg} \mathrm{kg}^{-1}\right)\end{array}$ & $\begin{array}{c}\mathrm{P} \\
\left(\mathrm{mg} \mathrm{kg}^{-1}\right)\end{array}$ & $\begin{array}{c}\mathrm{K} \\
\left(\mathrm{mg} \mathrm{kg}^{-1}\right)\end{array}$ & $\begin{array}{c}\mathrm{CO}_{3} \\
(\%)\end{array}$ & $\begin{array}{c}\mathrm{Ce} \\
\left(\mathrm{dS} \mathrm{m}^{-1}\right)\end{array}$ & $\begin{array}{c}\text { PSI } \\
(\%)\end{array}$ & $\begin{array}{c}\text { RAS } \\
(\%)\end{array}$ \\
\hline $0-30$ & 5.50 & 12.0 & 1648.0 & 5.30 & 0.67 & 1.79 & 2.09 \\
$30-60$ & 31.50 & 9.0 & 1848.0 & 6.80 & 1.20 & 1.76 & 2.07 \\
$60-90$ & 11.00 & 24.0 & 1131.0 & 8.66 & 1.84 & 2.48 & 2.58 \\
\hline $\begin{array}{r}\mathrm{NO}_{3}=\text { nitratos; } \\
\text { porciento de sodio intercambiable; }\end{array}$
\end{tabular}

Cuadro 2. Características físicas del suelo del sitio experimental.

\begin{tabular}{ccccccccc}
\hline $\begin{array}{l}\text { Prof. } \\
(\mathrm{cm})\end{array}$ & $\begin{array}{c}\text { MO } \\
(\%)\end{array}$ & $\begin{array}{c}\text { Arena } \\
(\%)\end{array}$ & $\begin{array}{c}\text { Limo } \\
(\%)\end{array}$ & $\begin{array}{c}\text { Arcilla } \\
(\%)\end{array}$ & Textura & $\begin{array}{c}\text { CC } \\
(\%)\end{array}$ & $\begin{array}{c}\text { PMP } \\
(\%)\end{array}$ & $\begin{array}{c}\text { Da } \\
\left(\mathrm{g} \mathrm{cm}^{-3}\right)\end{array}$ \\
\hline $0-30$ & 1.08 & 38.0 & 38.0 & 24.0 & Franco arcilloso & 30.6 & 15.9 & 1.3 \\
$30-60$ & 0.67 & 38.0 & 38.0 & 24.0 & Franco arcilloso & 30.0 & 14.7 & 1.2 \\
$60-90$ & 0.33 & 41.4 & 35.4 & 23.2 & Franco & 29.3 & 14.4 & 1.3 \\
\hline
\end{tabular}

MO = materia orgánica; $\mathrm{CC}=$ capacidad de campo; PMP = punto de marchitez permanente; $\mathrm{Da}=$ densidad aparente.

\begin{tabular}{|c|c|c|}
\hline Tratamiento & Núm. de riegos & Humedad aprovechable residual del suelo (\%) \\
\hline 1 & 1 & Riego de presiembra \\
\hline 2 & 2 & 0 \\
\hline 3 & 3 & 12 \\
\hline 4 & 4 & 24 \\
\hline 5 & 5 & 36 \\
\hline 6 & 6 & 48 \\
\hline 7 & 7 & 60 \\
\hline
\end{tabular}

Cuadro 4. Ecuaciones características de la humedad del suelo.

\begin{tabular}{ccc}
\hline Profundidad $(\mathrm{cm})$ & Ecuaciones & $\mathrm{R}^{2}$ \\
\hline $0-30$ & $\mathrm{~T}=295656811.6 \mathrm{Ps}^{-6.104}$ & 0.98 \\
$30-60$ & $\mathrm{~T}=60154058.9 \mathrm{Ps}^{-5.56}$ & 0.98 \\
$60-90$ & $\mathrm{~T}=97333071.9 \mathrm{Ps}^{-5.68}$ & 0.98 \\
\hline $\mathrm{T}=$ tensión de humedad del suelo (atm); Ps $=$ contenido de humedad del suelo (\%).
\end{tabular}


Las variables de control fueron: contenido de agua en el suelo, expresado como humedad aprovechable residual (\%) y como tensión de humedad o potencial mátrico (MPa), y cantidad o lámina de agua consumida por cada tratamiento $(\mathrm{cm})$. Las variables respuesta fueron: rendimiento de grano $\left(\mathrm{t} \mathrm{ha}^{-1}\right)$, y la productividad del agua estimada como la relación entre el rendimiento de grano y la cantidad de agua consumida $\left(\mathrm{kg} \mathrm{m}^{-3}\right)$.

\section{RESULTADOS Y DISCUSIÓN}

El Cuadro 5 muestra los regímenes de riego correspondientes a los tratamientos aplicados a la canola durante su ciclo vegetativo, que duró $145 \mathrm{~d}$. El número de riegos aplicados al cultivo varió de 1 a 7 ; el consumo de agua de 14.9 a $60.1 \mathrm{~cm}$; el contenido de agua en el suelo expresado como humedad aprovechable residual al momento del riego, de 0.0 a $56.2 \%$, y su equivalente en tensión de humedad de -1.52 a $-0.26 \mathrm{MPa}$. Se considera que el rango de variación del régimen de humedad del suelo fue amplio, ya que el nivel de abatimiento máximo observado en el caso del tratamiento más seco rebasó el punto de marchitez permanente $(-1.5 \mathrm{MPa})$, que es cuando se agota por completo la humedad aprovechable del suelo.

\begin{tabular}{cccc} 
Cuadro 5. Régimen de riego en los tratamientos ensayados. \\
\hline Tratamiento & $\begin{array}{c}\text { Lámina } \\
\text { consumida } \\
(\mathrm{cm})\end{array}$ & $\begin{array}{c}\text { Humedad } \\
\text { aprovechable } \\
\text { residual }(\%)\end{array}$ & $\begin{array}{c}\text { Tensión de } \\
\text { humedad del } \\
\text { suelo }(\mathrm{MPa})\end{array}$ \\
\hline 1 & 14.9 & 0.0 & -1.52 \\
2 & 28.1 & 8.1 & -1.27 \\
3 & 34.8 & 19.9 & -1.00 \\
4 & 43.0 & 34.4 & -0.85 \\
5 & 49.1 & 35.6 & -0.64 \\
6 & 57.4 & 47.1 & -0.35 \\
7 & 60.1 & 56.2 & -0.26 \\
\hline
\end{tabular}

\section{Rendimiento de grano}

El rendimiento varió de 1.02 a $3.42 \mathrm{t} \mathrm{ha}^{-1}$ (Figura 1), rango similar al observado por Nielsen (1997) de 0.54 a $3.41 \mathrm{t} \mathrm{ha}^{-1}$ en un estudio de la respuesta de la canola al estrés hídrico efectuado en condiciones de riego en Colorado, Estados Unidos. En cambio, el rango de rendimiento observado es mayor que los rangos de 0.17 a 2.80 y de 2.80 a $2.58 \mathrm{t} \mathrm{ha}^{-1}$ reportados en los estados de Minnesota y Wisconsin, respectivamente (Oplinger et al., 2008), a partir de estudios realizados en zonas de temporal secano donde no se cubrieron en totalidad las necesidades hídricas de la canola.

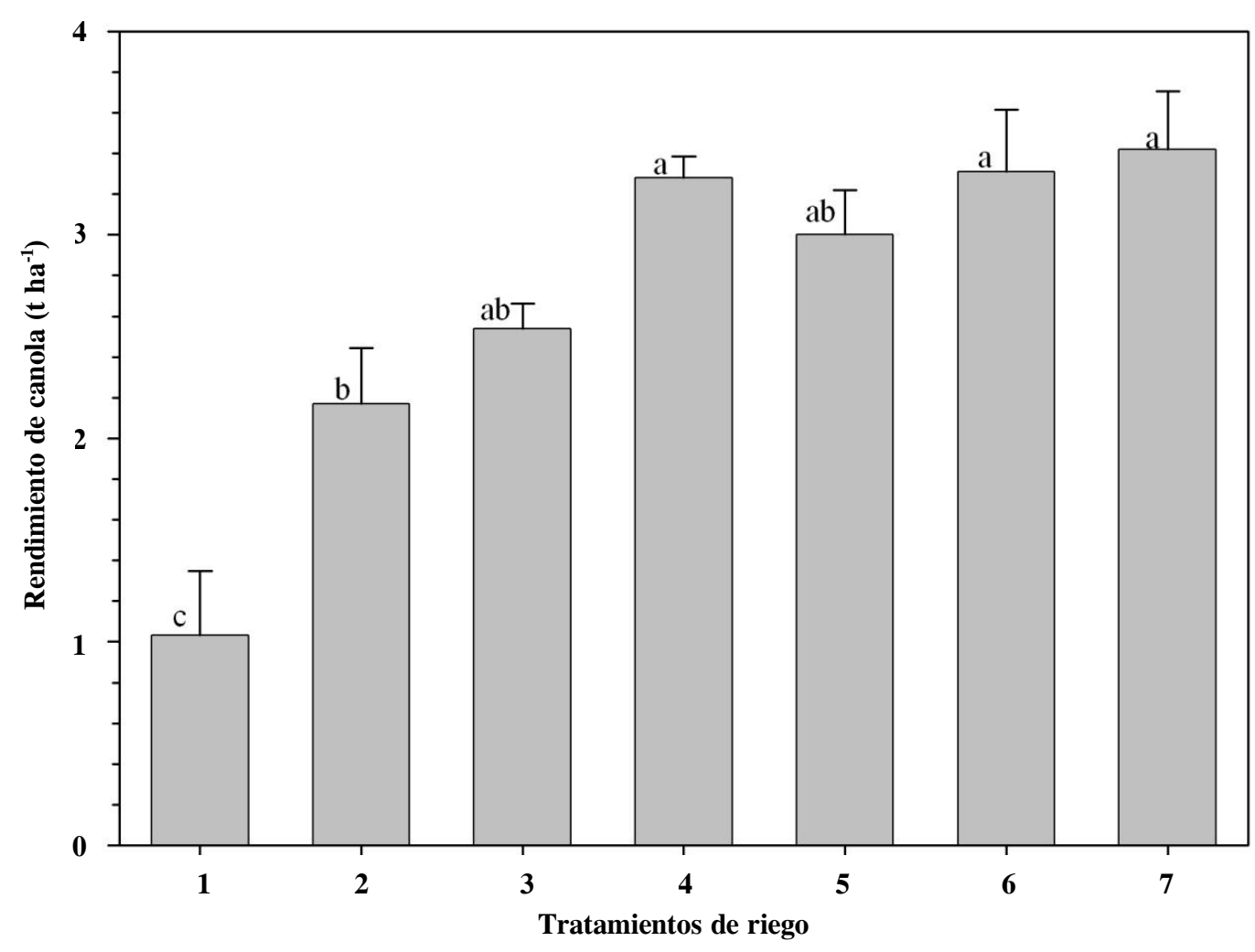

Figura 1. Rendimiento de grano de la canola, por tratamiento. Barras con las mismas letras son estadísticamente iguales (Tukey, 0.05). $1=$ riego de presiembra; $2=0 ; 3=12 ; 4=24 ; 5=36 ; 6=48 ; 7=60 \%$ de la humedad aprovechable residual del suelo. 
De acuerdo con el análisis de varianza practicado (Cuadro 6) y la prueba de comparación de medias de Tukey $(\alpha=0.05)$, los Tratamientos $4,5,6$ y 7 produjeron los rendimientos de grano más altos, con valores de 3.0 a $3.42 \mathrm{t} \mathrm{ha}^{-1}$, valores que fueron estadísticamente iguales entre sí $(p=0.05)$ y que se obtuvieron al aplicar el riego a niveles de tensión de humedad de -0.85 a -0.26 $\mathrm{MPa}$ durante el desarrollo de la canola. Los rendimientos más bajos de 1.02, 2.17 y $2.54 \mathrm{t} \mathrm{ha}^{-1}$ se obtuvieron con los tratamientos 1, 2 y 3 (Figura 1), cuando el cultivo se regó a tensiones de humedad de $-1.52,-1.27$ y $-1.0 \mathrm{MPa}$, respectivamente.

\section{Productividad del agua}

La productividad del agua observada para los diferentes tratamientos de riego varió de 0.57 a $0.77 \mathrm{~kg} \mathrm{~m}^{-3}$, de manera similar al rango de 0.22 a $0.71 \mathrm{~kg} \mathrm{~m}^{-3}$ reportado por Nielsen (1997). Sin embargo, no hubo diferencias significativas entre tratamientos para esta variable (Cuadro 6).

Cuadro 6. Cuadrados medios del análisis de varianza y prueba de $\mathbf{F}$ para rendimiento de grano y productividad del agua en canola.

\begin{tabular}{lccc}
\hline FV & gl & $\begin{array}{c}\text { Rendimiento de } \\
\text { grano }\left(\mathrm{t} \mathrm{ha}^{-1}\right)\end{array}$ & $\begin{array}{c}\text { Productividad del } \\
\text { agua }\left(\mathrm{kg} \mathrm{m}^{-3}\right)\end{array}$ \\
\hline Bloques & 3 & 0.167 & 0.0294 \\
Tratamientos & 6 & $2.878^{* *}$ & 0.0395 \\
Error & 18 & 0.233 & 0.0361 \\
CV $(\%)$ & & 18.1 & 25.7 \\
\hline
\end{tabular}

$\mathrm{FV}=$ fuente de variación; $\mathrm{gl}=$ grados de libertad; $* *=\mathrm{P} \leq 0.01 ; \mathrm{CV}$ $=$ coeficiente de variación.

\section{Función de producción del régimen de humedad del suelo}

Por medio del procedimiento de análisis de regresión múltiple (SAS Institute, 1999), se ajustaron los datos del rendimiento de grano de la canola como variable dependiente, y la humedad aprovechable residual del suelo al momento del riego como variable independiente. El mejor ajuste se logró con un modelo lineal de segundo orden (Ec. 1).

$$
\begin{gathered}
R g=1.21828+0.08639 \text { HAR }-0.00086389 H_{A R}^{2} \quad \text { (Ec. 1) } \\
R^{2}=0.79 ; \quad C V=16 \%
\end{gathered}
$$

Donde HAR representa la humedad aprovechable residual del suelo al momento del riego (\%), y $\mathrm{Rg}$ el rendimiento de grano de la canola $\left(\mathrm{t} \mathrm{ha}^{-1}\right)$.

De acuerdo con la teoría de máximos y mínimos del cálculo diferencial e integral (Martínez y Castillo, 1987), se deduce que el rendimiento de grano máximo de $3.38 \mathrm{t}$ ha $^{-1}$ se logra cuando se riega hasta que el suelo alcanza 50
$\%$ de la humedad aprovechable residual promedio, equivalente a $-0.22 \mathrm{MPa}$ de tensión de humedad. Sin embargo, el análisis numérico de la pendiente de la función de producción muestra que si la humedad aprovechable se reduce a $35 \%$, el rendimiento de grano obtenido es $3.18 \mathrm{t} \mathrm{ha}^{-1}$, es decir, disminuye sólo $5.7 \%$ con respecto al rendimiento máximo, debido a que en dicho rango de la humedad aprovechable la pendiente de la función tiene una tasa de cambio pequeña.

Estos resultados sugieren que los riegos de la canola deben aplicarse cuando la humedad aprovechable residual en el suelo disminuya hasta $35 \%$, como límite. En otros suelos, la tensión de humedad o potencial mátrico promedio a $90 \mathrm{~cm}$, no debería disminuir por abajo de $-0.74 \mathrm{MPa}$ entre un riego y otro, ya que la tensión de humedad representa mejor el efecto del déficit hídrico del suelo sobre el cultivo que la humedad aprovechable o contenido de agua, porque es una variable con mayor grado de extrapolabilidad a suelos con diferente textura y capacidad de retención de agua.

\section{Función de producción del consumo de agua}

La relación entre el rendimiento de grano $(\mathrm{Rg})$ y la lámina de agua consumida (Lc) también se ajustó mejor a un modelo lineal de segundo orden (Figura 2). Según este modelo, el rendimiento aumenta con una tasa o pendiente que disminuye progresivamente a partir de los $15 \mathrm{~cm}$ de lámina y hasta $60 \mathrm{~cm}$ de lámina donde la pendiente es cero, y alcanza su valor máximo de $3.35 \mathrm{t} \mathrm{ha}^{-1}$. De manera similar a la función de producción del régimen de humedad, si la lámina de agua se reduce de 60 a $48 \mathrm{~cm}$, el rendimiento de grano disminuye de 3.35 a $3.18 \mathrm{t} \mathrm{ha}^{-1}$, es decir, una relación de sólo $5.1 \%$.

En el rango de lámina de agua de 25 a $50 \mathrm{~cm}$, el rendimiento aumenta de 1.94 a $3.23 \mathrm{t} \mathrm{ha}^{-1}$, rango superior al aumento de 0.74 a $2.70 \mathrm{t} \mathrm{ha}^{-1}$ obtenido por Nielsen (1997) en Colorado, Estados Unidos, quien reportó un modelo lineal de ajuste para el rendimiento de grano de la canola en ese rango de lámina de agua. Tales diferencias se atribuyen a factores edafoclimáticos múltiples distintos al riego que restringen el ámbito de cobertura de las funciones de producción a un nivel regional, que para el caso del presente estudio sería las regiones áridas del norte de México. 


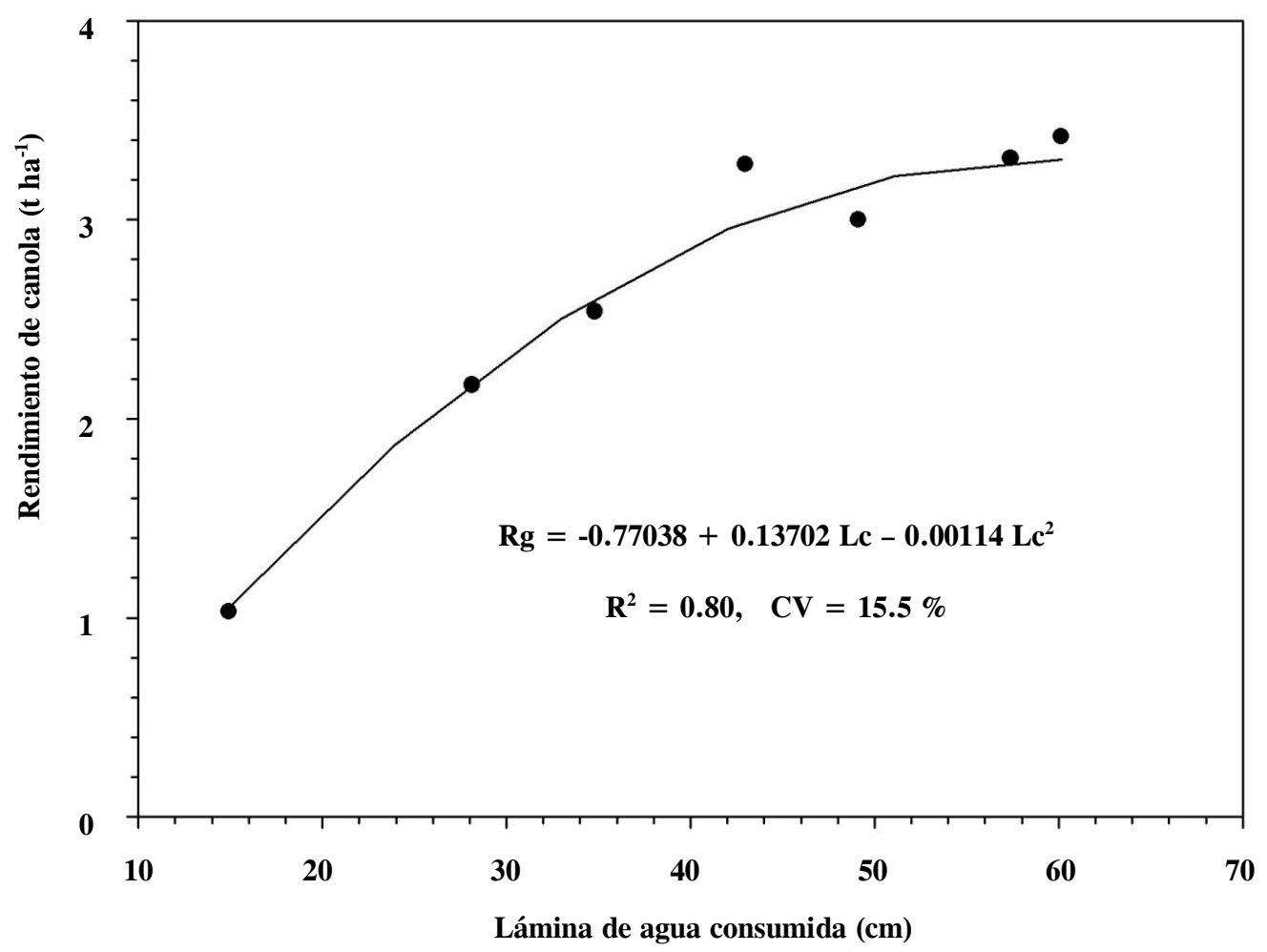

Figura 2. Función de producción del consumo de agua. Cada punto $(\cdot)$ representa la media de cuatro repeticiones; $\mathbf{R g}=$ rendimiento de grano de canola; $L c=$ lámina de agua consumida; $C V=$ coeficiente de variación; $R^{2}=$ coeficiente de determinación.

\section{CONCLUSIONES}

De acuerdo con las funciones de producción generadas, el rendimiento de grano de canola presenta una respuesta no lineal tanto al déficit hídrico del suelo como al consumo de agua. Tales funciones permiten inferir que la canola puede llegar a tener un rendimiento máximo de 3.3 $\mathrm{t} \mathrm{ha}^{-1}$, con una productividad del agua de $0.77 \mathrm{~kg} \mathrm{~m}^{-3}$. Este máximo se logra con una lámina de agua consumida de $60 \mathrm{~cm}$, aplicada con riegos cuando la humedad aprovechable residual en el suelo baja a $50 \%$, equivalente a una tensión de humedad de $-0.32 \mathrm{MPa}$. Es posible lograr un rendimiento similar de $3.1 \mathrm{t} \mathrm{ha}^{-1} \mathrm{y}$ un importante ahorro de agua $\left(1200 \mathrm{~m}^{3} \mathrm{ha}^{-1}\right)$ si el consumo de ésta se reduce a $48 \mathrm{~cm}$, mediante riegos a $35 \%$ de la humedad aprovechable residual en el suelo, equivalente a una tensión de humedad de $-0.74 \mathrm{MPa}$.

Las funciones de producción del agua de riego aquí obtenidas representan herramientas de análisis útiles para pronosticar los niveles potenciales de producción y productividad de la canola en diferentes escenarios de disponibilidad de agua en las zonas áridas del norte de México.

\section{BIBLIOGRAFÍA}

Al-Ghobari H M (2000) Estimation of reference evapotranspiration for southern region of Saudi Arabia. Irrig. Sci. 19:81-86.

Castellanos J Z, S X Uvalle B, A Aguilar S (2000) Manual de Interpretación de Análisis de Suelos y Aguas. 2a ed. Colección INCAPA. San Miguel de Allende, Gto. 226 p.

Gan Y, S V Angadi, H Cutforth, D Potts, V V Angadi, C L McDonald (2004) Canola and mustard response to short periods of temperature and water stress at different developmental stages. Can. J. Plant Sci. 84:697-704.

García E (1970) Modificaciones al Sistema de Clasificación Climática de Köppen (para adaptarlo a las condiciones de la República Mexicana). Instituto de Geografía de la UNAM, para la Comisión de Estudios del Territorio Nacional. 235 p.

Johnston A M, D L Tanaka, P R Miller, S A Brandt, D C Nielsen, G P Lafond, $\mathbf{N} \mathbf{R}$ Riveland (2003) Oilseed Crops for Semiarid Cropping Systems in the Northern Great Plains. Agron. J. 94:231-240.

Kassam A H, D Molden, E Fereres, J Doorenbos (2007) Water productivity: science and practice-introduction. Irrig. Sci. 25:185-188.

Martínez G A, A Castillo M (1987) Teoría de la Regresión con Aplicaciones Agronómicas. Ed. del Colegio de Postgraduados. Chapingo, Estado de México. 318 p. 
Mendoza M S F, M A Inzunza I, M M Villa C, E A Catalán V, A Román L, J Martínez S, H Macías R, C Potisek T (2004) Respuesta del chile jalapeño al acolchado plástico y niveles de humedad con riego por goteo (cintilla). In: XV Semana Internacional de Agronomía FAZ-UJED. J J Martínez R, S Berumen P, J Martínez T, A Martínez R (eds). 8-12 sept. Venecia, Durango. pp:489-494.

Nielsen D C (1997) Water use and yield of canola under dryland conditions in the Central Great Plains. J. Prod. Agric. 10:307-313.

Oplinger E S, L L Hardman, E T Gritton, J D Doll, K A Kelling (2008) Canola (Rapeseed). Alternative Field Crops Manual. Disponible en: http://www.hort.purdue.edu/newcrop/afcm/ canola.html (Marzo 2008).

Richards L A (1977) Diagnóstico y rehabilitación de suelos salinos y sódicos. 6a ed. Ed. Limusa. México. 172 p.

Rife C L, H Zeinali (2003) Cold tolerance in oilseed rape over varying acclimation durations. Crop Sci. 43:96-100.

Sánchez C I (2005) Fundamentos para el aprovechamiento integral del agua. Libro Científico No. 2. Instituto Nacional de
Investigaciones Forestales Agrícolas y Pecuarias, Centro Nacional de Investigación Disciplinaria en Relaciones Agua Suelo Planta Atmosfera. Gómez Palacio, Durango, México. $272 \mathrm{p}$.

Sánchez C I, H Macías R, G González C, F Mendoza M, M A Inzunza I, J Estrada A (2006) Planeación multiobjetivo en los distritos de riego de México. Aplicación de un sistema de auxilio para la toma de decisiones. Ing. Hidrául. Méx. 21:101-111.

SAS Institute Inc (1999) SAS/STAT User's guide, Version 6. 4th ed. Volume 2 Cary, NC. USA. 675p.

Villa C M, E A Catalán V, A Román L, M A Inzunza I, S F Mendoza M, J J Duarte R, A D Báez, G B L Gómez L (2007) Parámetros fisiotécnicos y requerimientos agroclimáticos de la canola (Brassica napus 1.). Agrofaz 9:13-19.

Willenborg C J, R H Gulden, E N Johnson, S J Shirtliffe (2004) Germination characteristics of polymer-coated canola (Brassica napus L.) seeds subjected to moisture stress at different temperatures. Agron. J. 96:786-791. 